\title{
Clinical Performances and Biological Features of Clear Aligners Materials in Orthodontics
}

\author{
Monica Macrì *, Giovanna Murmura, Giuseppe Varvara, Tonino Traini and Felice Festa \\ Department of Innovative Technologies in Medicine and Dentistry, University "G. D'Annunzio" of Chieti-Pescara, Chieti, Italy
}

In recent years, aesthetic concerns regarding orthodontic appliances have significantly increased due to the growing number of adult patients undergoing orthodontic therapy. Clear removable aligners have seen growing popularity as an aesthetic and comfortable alternative to traditional fixed appliances. Clear aligner therapy also appears more respectful of the patient's periodontal health; in fact, clear aligners allow the patients to maintain proper daily oral hygiene thanks to being removable. Among the parameters that affect the clinical efficacy of aligners, the material employed for their manufacturing plays a key role. The present paper aims to review the most used materials in manufacturing clear aligners, focusing on their clinical and mechanical performances, according to the current

OPEN ACCESS

Edited by: Michele Tepedino,

University of L'Aquila, Italy

Reviewed by:

Roberto Savignano,

Loma Linda University, United States Miguel Ángel Garcés-Villalá, Catholic University San Antonio of Murcia, Spain

*Correspondence: Monica Macri m.macri@unich.it

Specialty section: This article was submitted to Biomaterials,

a section of the journal

Frontiers in Materials

Received: 20 November 2021 Accepted: 03 January 2022 Published: 11 February 2022

Citation:

Macrì M, Murmura G, Varvara G, Traini $T$ and Festa $F$ (2022) Clinical Performances and Biological Features

of Clear Aligners Materials in Orthodontics.

Front. Mater. 9:819121. doi: 10.3389/fmats.2022.819121 state of literature. Furthermore, biological features of the different materials are also examined regarding their effects on dental and periodontal tissues, oral mucosa, and potential systemic effects.

Keywords: thermoplastic materials, physical properties, mechanical properties, clear aligner, clinical performances, biocompatibility

\section{INTRODUCTION}

The idea to employ clear overlay orthodontic appliances to move progressively misaligned teeth was introduced in 1946 by Kesling, who described how consecutive tooth movement is possible by utilising positioners produced from setup models (Kesling, 1945; Kesling, 1946).

In the last decades, thanks to the introduction and spread of CAD/CAM technologies in dentistry, the use of clear removable splints for orthodontic purposes has received a great impulse.

The first digitally designed and manufactured aligners system was the Invisalign system, a series of removable polyurethane aligners launched in 1998 by Align Technology (Santa Clara, CA, United States). In 1999 it was presented at the American Congress of Orthodontists. In 2001 it was introduced in Europe (Galan-Lopez et al., 2019). Currently, it is one of the most used clear aligners systems worldwide (Galan-Lopez et al., 2019; Nemec et al., 2020).

In recent years, the increase of adult patients undergoing orthodontic treatments has led to the widespread clear aligner therapy (CAT), considered a valid alternative to conventional fixed appliances for its aesthetic features and comfort (Rosvall et al., 2009; Fujiyama et al., 2014). Literature has reported various potential advantages correlated to CAT, such as the maintenance of better oral hygiene and periodontal health (Rossini et al., 2015; Lu et al., 2018; $\mathrm{Wu}$ et al., 2020), reduction in the amount and incidence of root resorption following orthodontic therapy (Yi et al., 2018; Li et al., 2020), the improvement of TMD-related pain and headache (Festa et al., 2021). Nowadays, clear aligner systems are produced 
TABLE 1 | Chemical (molecular) structure of some widely mass-marketed aligners and retainers.

\begin{tabular}{|c|c|c|}
\hline Aligner & Material & Manufacturer \\
\hline Biolon & PET-G (Polyethylene terephthalate glycol) & Dreve dentamid GmbH. Unna, Germany \\
\hline Duran & PET & Scheu dental, Iserlohn, Germany \\
\hline EasyDU & PET (PFb/PFc) & BenQ Co., Taipei, Taiwan \\
\hline F22 & Polyurethane & Sweden-Martina, Due Carrare, PD, Italy \\
\hline Invisalign & SmartTrack (multi-layer aromatic thermoplastic polyurethane) & Align Technology, Santa Clara, CA, United States \\
\hline MaxFlex & TPU & Maxflex Co., Taipei, Taiwan \\
\hline Nuvola & Polyethylene terephthalate glycol (PET-G) & GEO srl, Rome, RM, Italy \\
\hline Spark & Trugen (multi-layer polyurethane) & Ormco Orange, CA, United States \\
\hline
\end{tabular}

worldwide by numerous companies, including leading brands in orthodontic products (Table 1).

The clinical efficacy of clear aligners can be affected by many factors. Undoubtedly, the properties of materials used to produce clear aligners are among the essential aspects in determining their mechanical and clinical features.

The present paper study reviews the most employed materials in the manufacturing of clear aligners, focusing on their mechanical performances and biological features about their effects on dental and periodontal tissues and oral mucosa.

\section{CLEAR ALIGNERS MATERIALS}

Materials employed to produce Clear Aligners can affect their clinical performances (Zhang et al., 2011; Lombardo et al., 2017). The type of material used depends on the manufacturing process. Aligners can be produced by moulding the material on physical models, derived from a virtual planning software through $3 \mathrm{D}$ printing, or generated directly by $3 \mathrm{D}$ printing, without physical models (Tartaglia et al., 2021).

Currently, since no approved photo polymerisable resin is suited for direct printing, only thermoformed aligners are commercialised and clinically employed (Tartaglia et al., 2021).

\subsection{Thermoplastic Materials}

According to their molecular structure, thermoplastic polymers can be classified into amorphous and semicrystalline polymers. Amorphous polymers have irregularly arranged molecular structures characterised by a low degree of molecular packing. Semicrystalline polymers, instead, contain both areas of uniformly and tightly packed chains (crystalline domains) and irregularly arranged areas (amorphous regions). Crystalline domains can be comparable to fillers in composite materials in these polymers, which confer hardness and rigidity. In general, amorphous polymers are softer, transparent, have low shrinkage, and have better impact resistance. Semicrystalline polymers are hard, opaque or translucent, have good chemical resistance and have a sharp melting point (Chalmers and Meier, 2008; Condò et al., 2021).

The most used polymers, individually or blended, for the production of transparent orthodontic aligners are polyester, polyurethane, and polypropylene (Zhang et al., 2011; Condò et al., 2021).

Among polyesters, polyethylene terephthalate (PET) and polyethylene terephthalate glycol (PETG), a non-crystallising amorphous copolymer of PET, are widely used in the production of clear aligners thanks to their excellent mechanical and optical properties (Dupaix and Boyce, 2005).

Polycarbonate (PC) is also employed for its durability, hardness and transparency (Zhang et al., 2011).

Thermoplastic polyurethane (TPU) an extremely versatile material, featuring several favourable properties such as excellent mechanical and elastomeric characteristics, chemical and abrasion resistance, adhesion properties, simplicity of machining (Frick and Rochman, 2004; Zhang et al., 2011).

Invisalign aligners, were initially produced of a single-layer of polyurethane, Exceed-30 (EX30). In 2013, EX30 was substituted by a new polymer, named Smart Track (LD30) a multilayer aromatic thermoplastic polyurethane/copolyester (Rossini et al., 2015; Li et al., 2020). According to the producer, the new material should provide the aligners with more elasticity and produce more constant forces, improving their clinical efficacy (Lombardo et al., 2017; Condò et al., 2021).

PC, PETG are classified as amorphous (Chen et al., 2011; Demirel et al., 2011). Depending on processing procedures, PET and TPU can have an amorphous or semicrystalline structure (Frick and Rochman, 2004; Condò et al., 2021).

\subsection{Polymers Blends}

Thermoplastic orthodontic devices should exert continuous and controlled forces to produce correct tooth movements (Zhang et al., 2011).

Mechanical properties of the polymers can be improved by mixing various types of them: polyester, polyurethane, and polypropylene are the most used materials in the polymer blends employed in manufacturing of clear aligners (Zhang et al., 2011; Condò et al., 2021).

Large number of studies on thermoplastic polymer blends has been produced in recent years: (Medellín-Rodríguez et al., 1998; Hwang et al., 1999; Poomali et al., 2008; Zhang et al., 2011; Ma et al., 2016; Seeger et al., 2018). Polymer blending has proven to be a viable way to improve the physical and chemical properties of 
polymers, thereby enhancing the clinical performances of aligners.

The blending ratio of polymers employed plays an essential role in determining the features of the blend. For example, blending PETG/PC/TPU at the 70/10/20 ratio showed the best mechanical properties compared to other blending ratios, providing sufficient and sustainable orthodontic forces than other commercialised products (Zhang et al., 2011). PETG/PC2858 blend, at 70/30 ratio, expressed the best combination of tensile strength, impact strength and elongation at break (Ma et al., 2016).

\subsection{D Printed Aligners}

According to some authors, 3 days printed aligners can offer several advantages compared to thermoformed ones. Direct 3D printing can avoid adverse effects of thermoforming processes, such as alteration of mechanical, dimensional and aesthetic characteristics of the material (Ryu et al., 2018), offering better geometric accuracy and precision, better fit, higher efficacy and mechanical resistance and reproducibility (Maspero and Tartaglia, 2020).

3D printing techniques utilizable to build directly printed aligners can be numerous such as selective laser sintering (SLS), laser sintering melting (SLM), stereolithography (SLA). However, 3D printing by photo-polymerisation of clear liquid resin seems to be the most suitable procedure (Tartaglia et al., 2021).

Material employed through 3D printing in orthodontics can be very different. Among those, we can find acrylonitrilebutadiene-styrene plastic, stereolithography materials (epoxy resins), polylactic acid, polyamide (nylon), glass-filled polyamide, silver, steel, titanium, photopolymers, wax, and polycarbonate (Prasad et al., 2018).

Various studies have investigated mechanical and biological properties of resins suitable for 3D printing of clear aligners (Nakano et al., 2019; Maspero and Tartaglia, 2020); however, at present, no polymerizable material has yet been approved for the production of directly printed aligners (Tartaglia et al., 2021).

\section{MECHANICAL AND PHYSICAL PERFORMANCES OF CLEAR ALIGNER MATERIALS}

Dental misalignment and malocclusions usually compromise patients' aesthetics and smiles, which negatively impacting oral hygiene and periodontal health. Nowadays, the main reason patients today decide to undergo orthodontic treatment is precisely the improvement of the aesthetics of the face, and in turn, aesthetics play an essential role in the choice of orthodontic treatment. An increasing number of adult and adolescent patients require orthodontic treatment using transparent aligners because it is effective and does not compromise the quality of life because it is invisible (Bucci et al., 2019; Tartaglia et al., 2021).
Another benefit of these aligners is that they are removable, so they do not compromise the patients' oral hygiene, and the result is more comfortable than fixed appliances (Tartaglia et al., 2021).

The treatment is based on a sequence of upper and lower transparent aligners that the patient wears $22 \mathrm{~h}$ /day and changes after 14 days (Lombardo et al., 2015).

Thermoplastic materials of the aligners are polymers with different characteristics that respond differently to various types of mechanical stress such as chewing, physical stress such as heat and chemicals stress such as colouring agents, salivary enzymes and mouthwashes (Lombardo et al., 2015; Ma et al., 2016).

The ideal aligner should have excellent transparency, low hardness, resilience, elasticity, resistance to mechanical stress and overtime and biocompatibility. Therefore, to improve the performance of aligners in orthodontic treatment, it is significant to investigate the properties characteristic of the materials and how they respond to various stresses and then develop the more performing ones, for example, combining them (Ma et al., 2016).

\subsection{Colour Stability and Transparency of Different Types of Clear Aligners Materials}

In light of the patient's aesthetic, the transparency of the aligner should remain stable approximately during the 2 weeks of treatment (Liu et al., 2016). However, the aligners' colour stability and transparency are affected by colouring drinks, ultraviolet radiation, and mouthwashes (Bernard et al., 2020).

The dentists always recommend that patients remove the aligners when eating or drinking anything (except water). Often, many patients ignore the doctor's requests and eat and drink with the aligners, undermining their transparency, which is the essential aesthetic characteristic of the resinous copolymer that composes them (Bernard et al., 2020). It has been found that about $50 \%$ of Americans do not remove aligners for eating and drinking (Liu et al., 2016).

Different studies aim to investigate the aligners' colour stability and transparency when exposed to colouring agents and saliva (Liu et al., 2016; Daniele et al., 2020). The materials examined were: multi-layered thermoplastic polyurethane with integrated elastomer (Smart Track); one material based on PETG (Erkodur), one material of copolyester (Essix ACE), two based on PET (Essix Plastic e Ghost aligner) and resinous polyurethane (Zendura).

Visual inspection about colour stability indicated that all types of aligners showed no colour change after the 12-h, in contact with colouring agent (wine, coffee, black tea, cola and nicotine) except for the Smart Track in coffee and red wine.

With the increase of the time to 7 days, all the aligners showed colour changes slightly, except for the immersion in the coffee solution and black tea, which created an important colour change for all brands after 7 days. show a perceivable colour variation. In this last case, Essix P., Gost Aligner, Essix $\mathrm{A}$ and Zendura samples exhibited a slight colour variation; 
TABLE 2 | Colour stability.

\begin{tabular}{|c|c|c|c|c|c|c|}
\hline Time after & Coffee & Red wine & Nicotine & Artificial saliva & Black tea* & Cola* \\
\hline \multicolumn{7}{|l|}{$12 \mathrm{~h}$} \\
\hline \multicolumn{7}{|l|}{ Smart track** } \\
\hline \multicolumn{7}{|l|}{ zendura } \\
\hline \multicolumn{7}{|l|}{ erkodur } \\
\hline \multicolumn{7}{|l|}{ Essix ace } \\
\hline \multicolumn{7}{|l|}{ Essix Plastic } \\
\hline \multicolumn{7}{|l|}{ Ghost aligner } \\
\hline \multicolumn{7}{|l|}{$7 \mathrm{gg}$} \\
\hline \multicolumn{7}{|l|}{ Smart track** } \\
\hline \multicolumn{7}{|l|}{ Zendura } \\
\hline \multicolumn{7}{|l|}{ erkodur } \\
\hline \multicolumn{7}{|l|}{ Essix Ace } \\
\hline \multicolumn{7}{|l|}{ Essix Plastic } \\
\hline \multicolumn{7}{|l|}{ Ghost aligner } \\
\hline \multicolumn{7}{|l|}{$14 \mathrm{gg}$} \\
\hline \multicolumn{7}{|l|}{ Smart Track** } \\
\hline \multicolumn{7}{|l|}{ Zendura } \\
\hline \multicolumn{7}{|l|}{ Erkodur } \\
\hline \multicolumn{7}{|l|}{ Essix Ace** } \\
\hline \multicolumn{7}{|l|}{ Essix Plastic } \\
\hline Ghost aligner & & & & & & \\
\hline
\end{tabular}

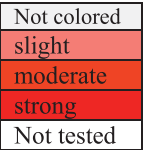

Grade of extrinsic coloration of aligners

*colouring agents tested only after $12 \mathrm{~h}$ and 7 days

**materials not tested after 14 days

while the Erkodur and SmartTrack samples display marked colour changes. Regarding the red wine, ST exhibit a substantial colour change after 7-day immersion.

Considering the immersion in the nicotine solution for 14 days, all the samples have only slightly colour changes; when immersed in red wine, they revealed enhanced colour variation, especially for Zendura and Essix P., while the other disks presented only perceivable variations (Table 2 ).

\subsection{The Influence of Thermoforming \\ Process}

Changes in material performances are made out also by the thermoforming process.

Various studies show how the transparency, hardness and thickness vary before and after the aligner's thermoforming process (Ryu et al., 2018; Bucci et al., 2019).

Ryu et al. study (Ryu et al., 2018) relates the thickness of the materials to the transparency before and after the thermoforming process and shows changes in four types of materials (two copolyester-based: Essix A + and Essix ACE another two pet-g based: Duran and ECligner).

The study has shown that thermoforming affects the material's transparency by decreasing it. After thermoforming, the transparency of the eCligner samples, 0.5 and $0.75 \mathrm{~mm}$ thickness, respectively, is remarkably reduced compared to that which characterises the Duran and Essix A + samples of the same thickness.

The Essix ACE sample, $0.75 \mathrm{~mm}$ thick, shows an important decrease in transparency compared to eCligner, with a thickness of $0.75 \mathrm{~mm}$.

Furthermore, the transparency of Duran and Essix A + samples (wall thickness) are significantly lower after the thermoforming compared to the pre-forming value, while no significant differences are seen, pre and post thermoforming, for the eCligner and Essix ACE samples.

The solubility in water and its absorption also affects the hardness of the material.

The hardness of all four materials did not show a significant difference compared to controls before thermoforming (Ryu et al., 2018). 


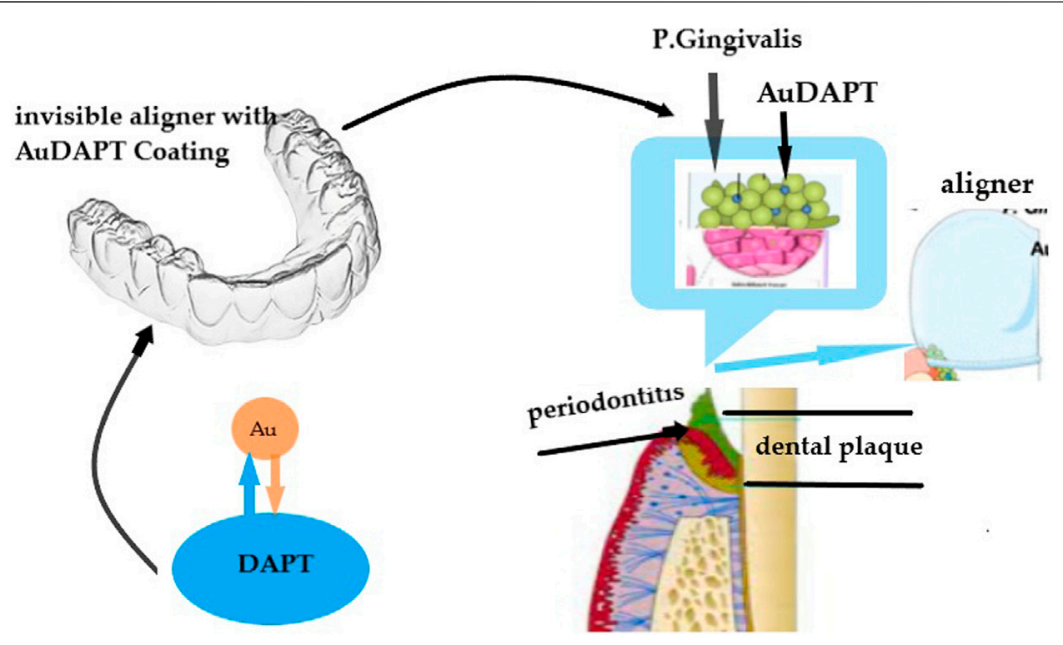

FIGURE 1 | Aligner with AuDAPT Coating.

In all four materials, there is an increase in the absorption ability of water after thermoforming. As for the solubility in water, there is a significant increase for Duran, Essix A + and Essix ACE samples, unlike what happens for eCligner samples.

However, The hardness of essix A and Essix Ace samples was less than the eCligner samples after thermoforming. Essix A + and Essix ACE samples showed higher surface hardness after thermoforming than before thermoforming (Ryu et al., 2018).

It has been seen through another study (Bucci et al., 2019) that investigated two types of aligners, one passive and one active in PET-G $0.75 \mathrm{~mm}$ film, that the thermoforming process can make changes in the thickness of the aligners.

After thermoforming, There are different thicknesses throughout the occlusal surface ranging from a minimum value of $0.38 \mathrm{~mm}$ to a maximum of $0.69 \mathrm{~mm}$. These values are lower than the 0.75 of the original PET-G sheets before being thermoformed.

Despite this variation, this does not affect the clinical performance of the devices.

Similar results are also obtained in the in vitro study of Dalaie et al. (Dalaie et al., 2021).

The study of Dalaie et al. investigated the thermomechanical properties of two PET-G aligners of two different thicknesses, 1 and $0.8 \mathrm{~mm}$, in response to thermoforming; it has been seen that in both types, the hardness decreases by about $7.6 \%$ after thermoforming, but there are no significant differences in hardness into the two types of aligners. The reduction of thickness decreases after thermoforming in both types of aligners, which justifies the reduction of flexural modulus (Dalaie et al., 2021). Comparing the flexural modulus of Duran with a thickness of 0.75 and Duran $1 \mathrm{~mm}$, the first modulus increases and the second decrease, meaning that with increasing thickness of the Pet-G sheets, the bending modulus decreases (Dalaie et al., 2021).

\subsection{The Influence of the Oral Environment}

In the oral cavity, the aligners are subject to humidity, salivary enzymes and temperature variations that can alter their shape and properties throughout time. However, they are also subjected to continuous and intermittent forces due to normal oral functions such as chewing, speaking, swallowing and parafunction such as clenching and grinding (Bucci et al., 2019). The temperature of the oral environment can rise to $57^{\circ}$ after taking a hot drink, and it can take several minutes to return to its original temperature. Such temperature increases can affect the mechanical properties of thermoplastics (Iijima et al., 2015). As evidence of this, there are several in vivo and in vitro studies (Ryokawa et al., 2006; Iijima et al., 2015; Bucci et al., 2019; Dalaie et al., 2021).

One in vitro study based its results on comparing eight different types of most used materials (PET-G, PC, PP, PUR, $\mathrm{A}+, \mathrm{C}+, \mathrm{PE}$ and EVA) from different manufacturers and placed them in contact with a solution that simulated the oral environment (Ryokawa et al., 2006).

It assessed that the elastic modules of Polycarbonate (PC), PET-G and Essix A + in the intraoral environment exhibited significant increases compared to the original samples; in reverse, those of polypropylene (PP), C +, polyethylene (PE) and Ethylene Vinyl Acetate (EVA)were significantly reduced. No significant changes were observed in the polyurethane (PUR).

On the other hand, an increase in thickness in all materials was also highlighted due to water absorption (Ryokawa et al., 2006).

The in vitro study by Dalaie (Dalaie et al., 2021) simulated the temperature variations that occur in the oral environment through intermittent thermocycles, $22 \mathrm{~h}$ per day for 14 days and studied how the hardness, thickness and flexural modulus of two aligners (in Pet-G foil with thicknesses of 1 and $0.8 \mathrm{~mm}$ ) vary in contact of the oral environment.

Significant variations in hardness were highlighted only in the $0.8 \mathrm{~mm}$ thick PET-G sheet; in the other $1 \mathrm{~mm}$ sheet, there were no significant ones. This increase in hardness can be attributed to changes in the crystalline and amorphous structures or the release of plasticisers after exerting intermittent thermal cycling.

The study aimed to investigate the effects of temperature variations on the shape memory properties of five 
TABLE 3 | Cytotoxicity of four of different thermoplastic materials for clear aligners. Cell viability (\%) = (optical density of test group/optical density of cellular control group) $\times$ 100. Cytotoxicity scored according to the classification of Ahrari et al. (from Martina et al., modified) (Ahrari et al., 2010; Martina et al., 2019).

\begin{tabular}{|c|c|c|c|}
\hline Brand & Composition & Cell viability (\%) & Cytotoxicity \\
\hline Biolon & Polyethylene terephthalate glycol (PETG) & $64.6 \pm 3.31$ & Slight \\
\hline Zendura & Polyurethane resin & $74.4 \pm 2.34$ & Slight \\
\hline SmartTrack & Multilayer aromatic thermoplastic polyurethane & $78.8 \pm 6.35$ & Slight \\
\hline Duran & Polyethylene terephthalate glycol (PETG) & $84.6 \pm 4.02$ & Slight \\
\hline
\end{tabular}

thermoplastic materials with different glass transition temperatures and different crystalline structures (Iijima et al., 2015). The materials selected are PET-G (Duran), polypropylene (Hardcast), polyurethane polymers (PU1 PU2 PU3) with three different glass transition temperatures.

The mechanical properties for each material were significantly reduced after 2,500 thermocycles, and a significant decrease is observed in Hardcast material (crystal plastic) with the highest Tg $\left(155.5^{\circ}\right)$ and PU 1 (crystalline or semicrystalline plastic) with the lower $\operatorname{Tg}\left(29.6^{\circ} \mathrm{C}\right)$. Duran $\left(73.3^{\circ}\right)$, PU $2\left(56.5^{\circ}\right)$ and PU3 $\left(80.7^{\circ}\right)$, with intermediate $\mathrm{Tg}$, exhibited more stable mechanical properties. Polyurethane polymers exhibited excellent shape memory undergoing the range of intraoral temperature variations.

The orthodontic strength produced by thermoplastic devices decreased for all materials with the gradual temperature change.

The in vivo study (Bucci et al., 2019) plays an essential role in the orthodontic history of materials because it considers the oral cavity's temperature variations and all the oral functions and parafunction to which the aligners are subjected.

A series of passive and active aligners formed by PET-G foil is used in this study, and patients were instructed to wear them 10 days for $22 \mathrm{~h}$ per day, and it was seen how their thickness changes after this time.

It was found that there were small reductions in the thickness of the aligner after 10 days but not significant as to affect the therapeutic performance, so the sheets of PeT-G have good stability in contact with the oral environment (Bucci et al., 2019).

\subsection{The Influence of Mechanical Stress and the Phenomenon of Stress Relaxation}

The aligners have an essential characteristic; they are viscoelastic, so they are in the middle between the properties of viscous and elastic materials. This means that their behaviour can vary significantly over time under load, even when inserted first and before any tooth movement.

Under constant loads, the deflection of the viscoelastic material increases over time, while at constant deflection, the loads decrease, and this phenomenon is called self-relaxation (Lombardo et al., 2017).

As already mentioned, the aligners placed in the oral cavity are subject to various stresses and intermittent loads in the long and short term. The phenomenon of stress relaxation reduces the forces exerted by the aligner placed in the mouth, at constant deflection and before the tooth begins to move. However, this depends on the characteristics of the material of aligners and the magnitude of the applied loads. It is essential to consider this reduction and quantify it to ensure that tooth movement occurs.

To examine the different mechanical characteristics of the materials, the study (Lombardo et al., 2017) investigated four types of materials, two single-layer materials based on PET-G and polyurethane and two multilayers.

After an initial resistance test, the samples were subjected to a constant load for $24 \mathrm{~h}$ in a humid environment and at a constant temperature, and the stress relaxation of the various materials was measured; the test was performed three times.

The monolayered aligners showed significant resistance to absolute stress and stress relaxation speed; the multi-layered ones instead showed a constant stress relaxation but an absolute stress resistance four times lower than the monolayered ones. In general, all the materials tested showed a significant relaxation to rapid stress in the first $8 \mathrm{~h}$, but $24 \mathrm{~h}$, it tended to plateau for some materials; for others, it decreased. The polyurethane-based monolayer aligner produced higher initial stress values and a high decay rate, the other one based on Pet-g showed the most significant stress relaxation rate during $24 \mathrm{~h}$. Multilayers showed lower stress relaxation rates and lowered initial stress values than monolayers (Lombardo et al., 2017).

\section{BIOCOMPATIBILITY OF MATERIALS USED AS ORTHODONTIC ALIGNERS}

According to a retrospective analysis by FDA (Food and Drug Administration) (Allareddy et al., 2017), the adverse clinical events reported during the use of active aligners were analysed. During a 10-years observation period, the most frequently reported adverse events are difficult breathing, sore throat, swollen throat, swollen tongue, hives and itchiness, anaphylaxis (Allareddy et al., 2017) adverse events during use of Invisalign Technology.

\subsection{Cytotoxicity of Materials}

The lack of scientific literature due to the few studies available and the contradictory results have kept the debate open about the existence of toxic effects related to the use of invisible aligners. Moreover, the increasing introduction of the new aligners has provided the need to test the cytotoxicity of the materials used by various manufacturers.

In vitro studies have evaluated the potential toxicity of thermoplastic materials used by different brands. Four different materials used for the aligners were evaluated: Duran (Germany), Biolon (Germany), Zendura (United States) and 
SmartTrack (United States). Human Gingival Fibroblasts (HGFs) are the cell lines often used to verify the biocompatibility of dental materials. Their use in vitro is recommended by the International Standards Organization (ISO) (Martina et al., 2019) because they constitute the main cell line present in the oral tissues and are the most exposed to the toxic effects of the materials of the aligners as they are in intimate contact with the periodontal tissues when they are in placeAmong the four tested materials, Biolon showed the highest toxic action on HGFs, followed by Zendura, SmartTrack, and finally, Duran proved to have the lowest toxic activity. In this study, it appears that all tested materials exhibit low in vitro toxicity on the tested cells. (Table 3 (Ahrari et al., 2010; Martina et al., 2019))

On the other hand, there are only two previous studies in which the cytotoxicity of clear aligner materials is tested. Eliades et al. evaluated the potential release of Bisphenol-A (BPA) from the materials used by the Invisalign technology (Eliades et al., 2009). The study shows the non-existence of estrogenic and toxic effects on HGFs, in contrast to the slight toxicity that emerged from the current study.

It has been shown that the chemical composition of thermoplastic Invisalign materials does not have the elements necessary to release BPA. Isocyanate and not BPS is the component that could develop potentially harmful effects on health (Alexandropoulos et al., 2015). Premaraj et al. (2014) found that the isocyanate in Invisalign aligners can trigger oral health effects. Allergic contact reactions have been reported following exposure to isocyanate. After contact with oral tissues, isocyanates react by rapidly binding to proteins and biomolecules, creating an immunogenic event that leads to a sensitization reaction in humans. These experiments have shown that the contact of the gingival epithelial cells with the plastic material in a saline-based environment determines an interruption of the membrane integrity, reduced metabolism and reduced cell-cell contact capacity. These Phenomena did not occur in artificial saliva. The results can play a protective role and reduce the effects of the plastic material of the allineators.

\subsection{Gold Nanoparticle-Modified Dental Aligner Used as Periodontal Therapy}

The correlation between the gingival pocket depth, gingival bleeding, periodontal disease and the presence of a type of bacteria in periodontal pockets have been widely demonstrated (De Iuliis et al., 2016). Appreciable changes in the oral microbiome, with higher anaerobic and facultative anaerobic bacteria, have been detected in patients wearing fixed orthodontic appliances (Kado et al., 2020). This effect is attributed to the fact that fixed appliances make dental hygiene procedures more difficult due to brackets and archwires. Conversely, the use of removable appliances may allow orthodontic patients to maintain adequate oral hygiene by allowing the standard procedures of brushing and flossing, which can be performed by easily removing the splint. Even though causing a significant change in the composition of the subgingival microbiome, CAT has shown to produce no changes in the relative presence of periodontal pathogens, at least in the first 3 months of therapy (Guo et al., 2018).

Several studies, referring to periodontal indexes such as plaque index, gingival index, probing depth, have reported that patients undergoing CAT have better periodontal health than those treated with fixed orthodontic appliances (Rossini et al., 2015; Lu et al., 2018; Wu et al., 2020).

A recent study showed that the aligners coated with gold nanoparticles (Zhang et al., 2020) determine favourable antibacterial activity against $\mathrm{P}$. gingivalis, one of the bacteria responsible for the onset of periodontal disease and other systemic diseases such as Alzheimer's disease. P. gigivalis has recently been identified in the brains of patients with Alzheimer's disease (Kaye et al., 2010; Dominy et al., 2019). This finding could lead to considerations of using aligners as a means of long-term drug delivery in patients with $\mathrm{P}$. gingivalis infection. The antibacterial action of gold nanoparticles (NPs) has opened up new research fields. Studies indicate the gold NPs exert their antibacterial action in different ways, such as reducing membrane potential, inhibiting ATPase activity, inhibiting the binding of ribosomes to tRNA. To evaluate the biocompatibility of AuDAPT, the haemolytic properties on mouse erythrocytes were tested. The results highlighted the absence of harmful irritative effects on the oral mucosa. Thus, AuDAPT can be applied for oral applications (Figure 1).

In conclusion, we can state that AuDAPT-coated aligners can perform antibacterial activity on P. gingivalis. Aligners coated with AuDAPT could slow biofilm formation showing favourable biocompatibility. This system could be used for the treatment of systemic infections related to periodontal disease.

\section{DISCUSSION}

In general, ideal properties of active components of orthodontic devices are considered large spring back, low stiffness, good formability, high stored energy, biocompatibility, and environmental stability (Kapila and Sachdeva, 1989). Even for clear aligners, the main features to be sought, in addition to high transparency and aesthetic stability, should be low hardness, good elasticity and resilience, resistance to ageing (Zhang et al., 2011; Ma et al., 2016). Physical and chemical characteristics of materials employed in the manufacturing of aligners are crucial in determining such features.

Nowadays, the material used to manufacture the aligners are polyethylene terephthalate glycol (PeT-G), polypropylene (PP), polycarbonate (PC), thermoplastic polyurethanes (TPU), ethylene-vinyl acetate (EVA) and many more (Lombardo et al., 2015).

This article investigates the performance of materials used in thermoplastic aligners, such as polyethylene terephthalate glycol (PeT-G), thermoplastic polyurethanes (TPU), polyethylene terephthalate PET.

Regarding aesthetic performances associated with colour stability and transparency, several studies show that the Ghost aligner based on pet-g material is more stable than the other in 
contact with colouring agents, especially coffee and red wine, after 7 days.

Colour stability and transparency of the aligner should remain stable approximately during the 2 weeks of treatment, but the studies and results are limited in this range of time.

Changes in material performances are made out also by the thermoforming process.

The thickness and hardness of aligners are significant; play a role in the magnitude of the forces delivered on the tooth by the device and, therefore, the performance of the aligners in orthodontic treatment.

The negative results of the thermoplastic process marked changes in the material's properties in response to the generation of heat used during thermoforming.

Ryu et al. (2018) show that the transparency decreases in all the samples after the thermoforming process, and the samples of Essix Ace copolyester-based (0.75 thickness) displays less transparency than the other samples after thermoforming of the same thickness.

After the thermoforming process, lower transparency values are recorded in the samples that had less thickness; for example, the transparency of Duran and Essix A + samples $(0.5 \mathrm{~mm}$ thickness) is significantly lower after the thermoforming compared to the pre-forming value, so this means that after thermoforming, the transparency decreases with decreasing thickness, since the thermoplastic material is deformed, resulting in a decrease in transparency, without compromise the aesthetics of the aligners themselves.

This finding contrasts with a previous study an increase in transparency with decreased thickness (Azhikannickal et al., 2012).

Regarding the hardness and water solubility, all thermoplastic materials show an increase in water absorption capacity following thermoforming and water solubility, except for the eCligner sample. (Ryu et al., 2018).

The hardness of the thermoplastic materials tested in this study increased after thermoforming. At the same time, the samples of Essix a and Essix Ace show a significant increase of hardness after thermoforming in the other samples is not so different from before thermoforming process. This means that the mechanical properties of the thermoplastic materials used for the production of CA should be studied for their clinical application, thinking to the thermoforming process.

This study contrasts with Delaie's study (Dalaie et al., 2021), which investigates the thermomechanical properties of two PET$\mathrm{G}$ aligners of two different thicknesses, 1 and $0.8 \mathrm{~mm}$, in response to seeing in both types, the hardness decreases by about $7.6 \%$ after thermoforming.

In the oral cavity, the aligners are subject to humidity, salivary enzymes and temperature variations that can alter their shape and properties throughout time. However, they are also subjected to continuous and intermittent forces due to normal oral functions such as chewing, speaking, swallowing and para functions such as clenching and grinding. (Bucci et al., 2019).

However, few in vivo studies can reproduce the natural oral environment in light of those facts. (Iijima et al., 2015). From this in vitro study (Ryokawa et al., 2006), the elastic modules of PC, PETG and A + in the intraoral environment showed significant increases compared to the original sample; in reverse, those of PP, $\mathrm{C}+, \mathrm{PE}$, and EVA were significantly reduced. No significant changes were observed in the PUR.

On the other hand, increased thickness in all materials was also highlighted due to water absorption.

The temperature variations that occur in the oral environment is replicated through intermittent thermocycles, $22 \mathrm{~h}$ per day for 14 days, and this study show how the hardness, thickness, and flexural modulus of two aligners based on PET-G of 1 and $0.8 \mathrm{~mm}$ of thickness vary in contact with this temperature variations (Dalaie et al., 2021).

Significant variations in hardness were highlighted only in the $0.8 \mathrm{~mm}$ thick PET-G sheet; in the other $1 \mathrm{~mm}$ sheet, there were no significant ones. The changes in the crystalline and amorphous structures or the release of plasticisers probably are linked to the increase in hardness after exerting thermal cycling (Dalaie et al., 2021).

Temperature variations linked to the oral environment also influenced the mechanical performance of materials, depending on different glass transitions (Iijima et al., 2015).

The materials such as Hardcast (polypropylene) with the highest $\operatorname{Tg}\left(155.5^{\circ}\right)$ and PU 1 (crystalline or semicrystalline plastic) with the lower $\mathrm{Tg}\left(29.6^{\circ} \mathrm{C}\right)$ were significantly decreased in mechanical properties after 2,500 thermo cycles, on the other hand, Duran, PU2 and PU3 which had intermediate $\mathrm{Tg}\left(75.3^{\circ} \mathrm{C}\right.$ for Duran, $56.5^{\circ} \mathrm{C}$ for PU 2 and $80.7^{\circ} \mathrm{C}$ for PU 3) showed stable mechanical properties (Iijima et al., 2015).

The orthodontic strength produced by devices decreased for all materials with the gradual temperature variation. (Ryokawa et al., 2006; Iijima et al., 2015; Bucci et al., 2019; Dalaie et al., 2021).

In the "in Vivo" study (Bucci et al., 2019), a series of passive and active aligners formed by PET-G foil was used, and patients were instructed to wear them 10 days for $22 \mathrm{~h}$ per day, and it was seen how their thickness changes after this time.

Ten days is a limited time, and usually in agreement with the doctor, it is decided to have them worn 14 days, the increase in the time they have to stay in the mouth can affect the loss of thickness.

Within the study's limits, the results show that there were small reductions in the thickness of the aligners after 10 days, but not significant as to affect the therapeutic performance, so the sheets of PeT-G have good stability in contact with the environment. (Bucci et al., 2019).

As already mentioned, the aligners placed in the oral cavity are subject to various stresses and intermittent loads in the long and short term. (Bucci et al., 2019).

To examine the different mechanical characteristics of the materials and their resistance to absolute stress and stress relaxation speed, a study investigates four types of materials, two single-layer materials based on PET-G and polyurethane and two multilayers in contact with a humid environment from 8 to 24 h (Lombardo et al., 2017).

The monolayered aligners showed significant resistance to absolute stress and stress relaxation speed; the multi-layered ones 
instead showed a constant stress relaxation but an absolute stress resistance four times lower than the monolayered ones. In general, all the materials tested showed a significant relaxation to rapid stress in the first 8 hours, but $24 \mathrm{~h}$ tended to plateau for some materials; for others, it decreased. (Lombardo et al., 2017).

The biocompatibility of clear aligners is still an open research field due to the lack of scientific literature and the few studies performed. The analysed studies evaluated in vitro toxicity of different thermoplastic materials used by the different brands. According to the experimental studies performed, all clear aligner materials exhibited mild cytotoxicity (Martina et al., 2019). Exposure of gingival epithelial cells to aligner plastics in a saline environment resulted in a reduction in membrane integrity, reduction in metabolic activity, and reduced intercellular contact of cell-cell junctions. The same effects were not found in artificial saliva. The results show how the plastic effects of active aligners can be neutralised or reduced in the presence of artificial saliva. Saliva could play an essential role in maintaining the integrity of epithelial cells (Premaraj et al., 2014).

It is essential to consider that it is difficult to compare the effects obtained in vitro with an in vivo environment, so we still do not have certainty regarding the absence of harmful effects for oral epithelium. In conclusion, we can state that the clear aligner materials showed only a low level of cytotoxicity, and the clinical use could be considered safe. Literature studies reveal that the use of clear aligners guarantees better maintenance of periodontal health than fixed appliances. The oral microbioma changes during orthodontic treatment with fixed appliances, increasing anaerobic bacteria and periodontal pathogens. This change is responsible for the transition from oral health to periodontitis

\section{REFERENCES}

Ahrari, F., Afshari, J. T., Poosti, M., and Brook, A. (2010). Cytotoxicity of Orthodontic Bonding Adhesive Resins on Human Oral Fibroblasts. Eur. J. Orthod. 32 (6), 688-692. doi:10.1093/ejo/cjq019

Alexandropoulos, A., Al Jabbari, Y. S., Zinelis, S., and Eliades, T. (2015). Chemical and Mechanical Characteristics of Contemporary Thermoplastic Orthodontic Materials. Aust. Orthod. J. 31 (2), 165-170.

Allareddy, V., Nalliah, R., Lee, M. K., Rampa, S., and Allareddy, V. (2017). Adverse Clinical Events Reported during Invisalign Treatment: Analysis of the MAUDE Database. Am. J. Orthod. Dentofacial Orthopedics 152 (5), 706-710. doi:10. 1016/j.ajodo.2017.06.014

Azhikannickal, E., Bates, P. J., and Zak, G. (2012). Laser Light Transmission through Thermoplastics as a Function of Thickness and Laser Incidence Angle: Experimental and Modeling. J. Manuf Sci. Eng. 134 (6). doi:10.1115/1.4007619

Bernard, G., Rompré, P., Tavares, J. R., and Montpetit, A. (2020). Colorimetric and Spectrophotometric Measurements of Orthodontic Thermoplastic Aligners Exposed to Various Staining Sources and Cleaning Methods. Head Face Med. 16 (1), 2. doi:10.1186/s13005-020-00218-2

Bucci, R., Rongo, R., Levatè, C., Michelotti, A., Barone, S., Razionale, A. V., et al. (2019). Thickness of Orthodontic clear Aligners after Thermoforming and after 10 Days of Intraoral Exposure: a Prospective Clinical Study. Prog. Orthod. 20 (1), 36. doi:10.1186/s40510-019-0289-6

Chalmers, J. M., and Meier, R. J. (2008). Molecular Characterization and Analysis of Polymers. Amsterdam: Elsevier.

Chen, L., Zhang, X.-l., Li, H.-y., Li, B., Wang, K., Zhang, Q., et al. (2011). Superior Tensile Extensibility of PETG/PC Amorphous Blends Induced via Uniaxial Stretching. Chin. J. Polym. Sci. 29 (1), 125-132. doi:10.1007/s10118-010-1006-6
(Kado et al., 2020). The qualitative and quantitative evaluation of plaque showed that periodontal health during treatment with CAT is better than treatment with fixed appliances. There is a significant decrease in periodontal indices (GI, PBI, BoP, PPD) (Rossini et al., 2015). The studies demonstrated that the periodontal status during orthodontic treatment with CAT is much superior to conventional fixed appliances (Wu et al., 2020).

Nanomaterials have recently been reported to exhibit antimicrobial activities above all gold, (NP) nanoparticles. Both in vitro and in vivo experiments were performed to evaluate the biocompatibility of the basis of AuDAPT. The antibacterial action of gold NPs is currently an active research field. Aligners coated with AuDAPT demonstrated favourable biocompatibility and an ability to slow biofilm formation (Zhang et al., 2020). It is thought that this method could be used to treat systemic infections related to periodontal disease. However, further information is needed because the microorganisms present in the oral cavity are more complex than a single type of bacterium. Investigation should be conducted in the future to simulate the biological environment and develop suitable methods for treating bacterial-related oral diseases through dental devices.

\section{AUTHOR CONTRIBUTIONS}

MM wrote the article. MM, FF, GM, TT, and GV researched the articles for the review and selected the appropriate studies. MM, GM, TT, GV, and FF analysed the studies for the review. All the authors read and approved the final manuscript.

Condò, R., Mampieri, G., Giancotti, A., Cerroni, L., Pasquantonio, G., Divizia, A., et al. (2021). SEM Characterization and Ageing Analysis on Two Generation of Invisible Aligners. BMC Oral Health 21 (1), 316. doi:10.1186/s12903-02101676-Z

Dalaie, K., Fatemi, S. M., and Ghaffari, S. (2021). Dynamic Mechanical and thermal Properties of clear Aligners after Thermoforming and Aging. Prog. Orthod. 22 (1), 15. doi:10.1186/s40510-021-00362-8

Daniele, V., Macera, L., Taglieri, G., Di Giambattista, A., Spagnoli, G., Massaria, A., et al. (2020). Thermoplastic Disks Used for Commercial Orthodontic Aligners: Complete Physicochemical and Mechanical Characterization. Materials 13 (10), 2386. doi:10.3390/ma13102386

De Iuliis, V., Ursi, S., Di Tommaso, L. M., Caruso, M., Marino, A., D’Ercole, S., et al. (2016). Comparative Molecular Analysis of Bacterial Species Associated with Periodontal Disease. J. Biol. Regul. Homeost Agents 30 (4), 1209-1215.

Demirel, B., Yaraş, A., and Elçiçek, H. (2011). Crystallization Behavior of PET Materials. Baü Fen Bil. Enst. Dergisi Cilt 13 (1), 26-35.

Dominy, S. S., Lynch, C., Ermini, F., Benedyk, M., Marczyk, A., Konradi, A., et al. (2019). Porphyromonas Gingivalis in Alzheimer's Disease Brains: Evidence for Disease Causation and Treatment with Small-Molecule Inhibitors. Sci. Adv. 5 (1), eaau3333. doi:10.1126/sciadv.aau3333

Dupaix, R. B., and Boyce, M. C. (2005). Finite Strain Behavior of Poly(ethylene Terephthalate) (PET) and Poly(ethylene Terephthalate)-Glycol (PETG). Polymer 46, 4827-4838. doi:10.1016/j.polymer.2005.03.083

Eliades, T., Pratsinis, H., Athanasiou, A. E., Eliades, G., and Kletsas, D. (2009). Cytotoxicity and Estrogenicity of Invisalign Appliances. Am. J. Orthod. Dentofacial Orthopedics 136, 100-103. doi:10.1016/j.ajodo.2009.03.006

Festa, F., Rotelli, C., Scarano, A., Navarra, R., Caulo, M., and Macrì, M. (2021). Functional Magnetic Resonance Connectivity in Patients with 
Temporomadibular Joint Disorders. Front. Neurol. 12, 629211. doi:10.3389/ fneur.2021.629211

Frick, A., and Rochman, A. (2004). Characterization of TPU-Elastomers by thermal Analysis (DSC). Polym. Test. 23, 413-417. doi:10.1016/j. polymertesting.2003.09.013

Fujiyama, K., Honjo, T., Suzuki, M., Matsuoka, S., and Deguchi, T. (2014). Analysis of Pain Level in Cases Treated with Invisalign Aligner: Comparison with Fixed Edgewise Appliance Therapy. Prog. Orthod. 15 (1), 64. doi:10.1186/s40510-014-0064-7

Galan-Lopez, L., Barcia-Gonzalez, J., and Plasencia, E. (2019). A Systematic Review of the Accuracy and Efficiency of Dental Movements with Invisalign ${ }^{\circledR}$. Korean J. Orthod. 49 (3):140-149.doi:10.4041/kjod.2019.49.3.140

Guo, R., Zheng, Y., Liu, H., Li, X., Jia, L., and Li, W. (2018). Profiling of Subgingival Plaque Biofilm Microbiota in Female Adult Patients with clear Aligners: a Three-Month Prospective Study. PeerJ 6, e4207. doi:10.7717/peer.4207

Hwang, S.-H., Jeong, K.-S., and Jung, J.-C. (1999). Thermal and Mechanical Properties of Amorphous Copolyester (PETG)/LCP Blends. Eur. Polym. J. 35 (8), 1439-1443. doi:10.1016/s0014-3057(98)00235-3

Iijima, M., Kohda, N., Kawaguchi, K., Muguruma, T., Ohta, M., Naganishi, A., et al. (2015). Effects of Temperature Changes and Stress Loading on the Mechanical and Shape Memory Properties of Thermoplastic Materials with Different Glass Transition Behaviours and crystal Structures. Eortho 37 (6), 665-670. doi:10.1093/ejo/cjv013

Kado, I., Hisatsune, J., Tsuruda, K., Tanimoto, K., and Sugai, M. (2020). The Impact of Fixed Orthodontic Appliances on Oral Microbiome Dynamics in Japanese Patients. Sci. Rep. 10 (1), 21989. doi:10.1038/s41598-020-78971-2

Kapila, S., and Sachdeva, R. (1989). Mechanical Properties and Clinical Applications of Orthodontic Wires. Am. J. Orthod. Dentofacial Orthopedics 96 (2), 100-109. doi:10.1016/0889-5406(89)90251-5

Kaye, E. K., Valencia, A., Baba, N., Spiro, A., 3rd, Dietrich, T., and Garcia, R. I. (2010). Tooth Loss and Periodontal Disease Predict Poor Cognitive Function in Older Men. J. Am. Geriatr. Soc. 58 (4), 713-718. doi:10.1111/j.1532-5415.2010.02788.x

Kesling, H. D. (1946). Coordinating the Predetermined Pattern and Tooth Positioner with Conventional Treatment. Am. J. Orthod. Oral Surg. 32, 285-293. doi:10.1016/0096-6347(46)90053-1

Kesling, H. D. (1945). The Philosophy of the Tooth Positioning Appliance. Am. J. Orthod. Oral Surg. 31 (6), 297-304. doi:10.1016/0096-6347(45)90101-3

Li, Y., Deng, S., Mei, L., Li, Z., Zhang, X., Yang, C., et al. (2020). Prevalence and Severity of Apical Root Resorption during Orthodontic Treatment with clear Aligners and Fixed Appliances: a Cone Beam Computed Tomography Study. Prog. Orthod. 21 (1), 1. doi:10.1186/s40510-019-0301-1

Liu, C.-L., Sun, W.-T., Liao, W., Lu, W.-X., Li, Q.-W., Jeong, Y., et al. (2016). Colour Stabilities of Three Types of Orthodontic clear Aligners Exposed to Staining Agents. Int. J. Oral Sci. 8 (4), 246-253. doi:10.1038/ijos.2016.25

Lombardo, L., Martines, E., Mazzanti, V., Arreghini, A., Mollica, F., and Siciliani, G. (2017). Stress Relaxation Properties of Four Orthodontic Aligner Materials: A 24-hour In Vitro Study. Angle Orthod. 87 (1), 11-18. doi:10.2319/113015-813.1

Lombardo, L., Arreghini, A., Maccarrone, R., Bianchi, A., Scalia, S., and Siciliani, G. (2015). Optical Properties of Orthodontic Aligners-Spectrophotometry Analysis of Three Types before and after Aging. Prog. Orthod. 16, 41. doi:10.1186/s40510-015-0111-z

Lu, H., Tang, H., Zhou, T., and Kang, N. (2018). Assessment of the Periodontal Health Status in Patients Undergoing Orthodontic Treatment with Fixed Appliances and Invisalign System. Medicine (Baltimore) 97 (13), e0248. doi: $10.1097 / \mathrm{md} .0000000000010248$

Ma, Y. S., Fang, D. Y., Zhang, N., Ding, X. J., Zhang, K. Y., and Bai, Y. X. (2016). Mechanical Properties of Orthodontic Thermoplastics PETG/PC2858 after Blending. Chin. J. Dent Res. 19 (1), 43-48. doi:10.3290/j.cjdr.a35696

Martina, S., Rongo, R., Bucci, R., Razionale, A. V., Valletta, R., and D'Antò, V. (2019). In Vitro cytotoxicity of Different Thermoplastic Materials for clear Aligners. Angle Orthod. 89 (6), 942-945. doi:10.2319/091718-674.1

Maspero, C., and Tartaglia, G. M. (2020). 3D Printing of Clear Orthodontic Aligners: Where We Are and where We Are Going. Materials 13 (22), 5204. doi: $10.3390 / \mathrm{mal} 3225204$

Medellín-Rodríguez, F. J., Phillips, P. J., Lin, J. S., and Avila-Orta, C. A. (1998). Triple Melting Behavior of Poly(ethylene Terephthalate Co-1,4-cyclohexylene Dimethylene Terephthalate) Random Copolyesters. J. Polym. Sci,part B:polym Phys. 36, 763-781. doi:10.1002/(sici)1099-0488(19980415)36:5<763:aid-polb4>3.0.co;2-m
Nakano, H., Kato, R., Kakami, C., Okamoto, H., Mamada, K., and Maki, K. (2019). Development of Biocompatible Resins for 3D Printing of Direct Aligners. J. Photopol. Sci. Technol. 32 (2), 209-216. doi:10.2494/photopolymer.32.209

Nemec, M., Bartholomaeus, H. M., H. Bertl, M., Behm, C., Ali Shokoohi-Tabrizi, H., Jonke, E., et al. (2020) Behaviour of Human Oral Epithelial Cells Grown on Invisalign SmartTrack Material ${ }^{\circledR}$ Materials 13 (23), 5311.doi:10.3390/ma13235311

Poomali, S., Sureshac, B., and Lee, J. H. (2008). Mechanical and Three-Body Abrasive Wear Behaviour of PMMA/TPU Blends. Mater. Sci. Eng. A 492 (1-2), 486-490. doi:10.1016/j.msea.2008.03.018

Prasad, S., Kader, N. A., Sujatha, G., Raj, T., and Patil, S. (2018). 3D Printing in Dentistry. J. 3D Printing Med. 2 (3), 89-91. doi:10.2217/3dp-2018-0012

Premaraj, T., Simet, S., Beatty, M., and Premaraj, S. (2014). Oral Epithelial Cell Reaction after Exposure to Invisalign Plastic Material. Am. J. Orthod. Dentofacial Orthopedics 145 (1), 64-71. doi:10.1016/j.ajodo.2013.09.011

Rossini, G., Parrini, S., Castroflorio, T., Deregibus, A., and Debernardi, C. L. (2015). Periodontal Health during clear Aligners Treatment: a Systematic Review. Eortho 37 (5), 539-543. doi:10.1093/ejo/cju083

Rosvall, M. D., Fields, H. W., Ziuchkovski, J., Rosenstiel, S. F., and Johnston, W. M. (2009). Attractiveness, Acceptability, and Value of Orthodontic Appliances. Am. J. Orthod. Dentofacial Orthopedics 135, 276-277. e1-276.e12; discussion 276-277. doi:10.1016/j.ajodo.2008.09.020

Ryokawa, H., Miyazaki, Y., Fujishima, A., Miyazaki, T., and Maki, K. (2006). The Mechanical Properties of Dental Thermoplastic Materials in a Simulated Intraoral Environment. Orthod. waves 65 (2), 64-72. doi:10.1016/j.odw.2006.03.003

Ryu, J.-H., Kwon, J.-S., Jiang, H. B., Cha, J.-Y., and Kim, K.-M. (2018). Effects of Thermoforming on the Physical and Mechanical Properties of Thermoplastic Materials for Transparent Orthodontic Aligners. Korean J. Orthod. 48 (5), 316-325. doi:10.4041/kjod.2018.48.5.316

Seeger, P., Ratfisch, R., Moneke, M., and Burkhart, T. (2018). Addition of ThermoPlastic Polyurethane (TPU) to Poly(methyl Methacrylate) (PMMA) to Improve its Impact Strength and to Change its Scratch Behavior. Wear 406-407, 68-74. doi:10.1016/j.wear.2017.11.024

Tartaglia, G. M., Mapelli, A., Maspero, C., Santaniello, T., Serafin, M., Farronato, M., et al. (2021). Direct 3D Printing of Clear Orthodontic Aligners: Current State and Future Possibilities. Materials 14 (7), 1799. doi:10.3390/ma14071799

Wu, Y., Cao, L., and Cong, J. (2020). The Periodontal Status of Removable Appliances vs Fixed Appliances. Medicine (Baltimore) 99 (50), e23165. doi:10.1097/md.0000000000023165

Yi, J., Xiao, J., Li, Y., Li, X., and Zhao, Z. (2018). External Apical Root Resorption in Non-extraction Cases after clear Aligner Therapy or Fixed Orthodontic Treatment. J. Dental Sci. 13 (1), 48-53. doi:10.1016/j.jds.2017.09.007

Zhang, M., Liu, X., Xie, Y., Zhang, Q., Zhang, W., Jiang, X., et al. (2020). Biological Safe Gold Nanoparticle-Modified Dental Aligner Prevents the Porphyromonas Gingivalis Biofilm Formation. ACS Omega 5 (30), 18685-18692. doi:10.1021/acsomega.0c01532

Zhang, N., Bai, Y., Ding, X., and Zhang, Y. (2011). Preparation and Characterization of Thermoplastic Materials for Invisible Orthodontics. Dent. Mater. J. 30 (6), 954-959. doi:10.4012/dmj.2011-120

Conflicts of Interest: The authors declare that the research was conducted in the absence of any commercial or financial relationships that could be construed as a potential conflict of interest.

Publisher's Note: All claims expressed in this article are solely those of the authors and do not necessarily represent those of their affiliated organizations, or those of the publisher, the editors and the reviewers. Any product that may be evaluated in this article, or claim that may be made by its manufacturer, is not guaranteed or endorsed by the publisher.

Copyright (๑) 2022 Macrì, Murmura, Varvara, Traini and Festa. This is an openaccess article distributed under the terms of the Creative Commons Attribution License (CC BY). The use, distribution or reproduction in other forums is permitted, provided the original author(s) and the copyright owner(s) are credited and that the original publication in this journal is cited, in accordance with accepted academic practice. No use, distribution or reproduction is permitted which does not comply with these terms. 